\title{
Plus de leur âge ? La sexualité des femmes de 50 ans dans les séries TV au début du $\mathrm{XXI}^{\mathrm{e}}$ siècle
}

Not acting their age? The sexuality of 50-year-old women in TV series in the early twenty-first century

\section{Mathieu Arbogast}

\section{OpenEdition}

\section{Journals}

Édition électronique

URL : http://journals.openedition.org/clio/12805

DOI : $10.4000 /$ clio. 12805

ISSN : 1777-5299

Éditeur

Belin

Édition imprimée

Date de publication : 1 décembre 2015

Pagination : 165-179

ISBN : 9782701194325

ISSN : 1252-7017

\section{Référence électronique}

Mathieu Arbogast, «Plus de leur âge ? La sexualité des femmes de 50 ans dans les séries TV au début du xxl ${ }^{e}$ siècle », Clio. Femmes, Genre, Histoire [En ligne], 42 | 2015, mis en ligne le 01 décembre 2018, consulté le 21 décembre 2020. URL : http://journals.openedition.org/clio/12805 ; DOI : https://doi.org/ $10.4000 /$ clio. 12805 


\section{Regard complémentaire}

\section{Plus de leur âge ? La sexualité des femmes de 50 ans dans les séries TV au début du XXI ${ }^{\mathrm{e}}$ siècle}

\section{Mathieu ARBOGAST}

En mai 2015, l'actrice américaine Maggie Gyllenhaal a expliqué au magazine The Wrap qu'elle venait d'être écartée d'un casting, parce qu'à 37 ans elle était jugée trop âgée pour interpréter l'amante d'un comédien de 55 ans $^{1}$. Différents travaux soulignent l'âgisme et le sexisme qui pèsent sur les actrices, à Hollywood en particulier ${ }^{2}$. Gyllenhaal soulève un point précis plus rarement abordé, qui pourrait aussi bien s'appliquer à une partie de la fiction audiovisuelle. Une femme jeune, un homme plus âgé, un nombre élevé d'années d'écart, voilà effectivement un schéma ordinaire dans les séries télévisées.

Les fictions télévisées sont une partie de l'expérience sociale. Ni reflet du monde social ni simples œuvres d'imagination ${ }^{3}$, elles occupent une place dans l'économie et dans les loisirs; nombre d'entre elles rassemblent des millions de spectateurs et ce sont elles qui sont en tête du classement des programmes les plus regardés. Dans la représentation des rapports de genre à l'écran, la différence d'âge dans le couple tient un rôle important. Un système asymétrique se dessine, celui d'un marché matrimonial organisé en fonction des

\footnotetext{
Waxman 2015.

2 Bazzini et al. 1997 ; Lincoln \& Allen 2004 ; Lauzen \& Dozier 2005 ; Jermyn 2013.

3 Coulomb-Gully 2012.
} 
préférences des hommes de tous âges, et où la jeunesse des femmes est un déterminant essentiel de leur désirabilité.

À partir d'un corpus de séries diffusées en France, on vérifiera d'abord la rareté des femmes de plus de 50 ans, alors que les hommes de ces âges y sont tout à fait présents. La jeunesse des femmes n'est pas seulement une valeur absolue (leur âge) mais aussi relative (l'écart avec leur partenaire masculin). On peut voir dans ces rapports d'âge contrastés la manifestation de la masculinité et de la féminité hégémoniques. La différence d'âge est une dimension-clé de l'asymétrie de genre, néanmoins quelques personnages joués par des femmes de plus de 50 ans ont une vie amoureuse échappant à ce schéma majoritaire. Dans quelle mesure ces représentations remettent-elles en question les normes de genre?

\section{Où sont les femmes (de 50 ans)?}

Des études quantitatives montrent que les comédiennes sont une minorité, depuis les débuts de la télévision. Joseph R. Dominick ${ }^{4}$ montre que, au cours de la période 1953-1977, les rôles féminins ont exceptionnellement atteint les $40 \%$ en début de période, pour ensuite osciller autour de $30 \%$ avec des étiages à $25 \%$ des rôles principaux. Minoritaires, ces femmes sont également concentrées dans les âges les plus jeunes.

\section{Une année de télévision en France}

Nous avons analysé un échantillon de séries diffusées en France. Les séries policières diffusées en début de soirée sur les quatre chaînes les plus regardées (TF1, France 2, France 3, M6) entre le 1er janvier et le 31 décembre 2010 ont été listées dans leur totalité. À une exception près, toutes ont eu au minimum deux «saisons ", jusqu'à 22 pour la plus pérenne ${ }^{5}$. Nous avons recensé tous les personnages principaux et

\footnotetext{
4 Dominick 1979.
}

5 Les épisodes commandés comme un lot et produits pour être diffusés une même année (scolaire ou civile) constituent une saison, que les intrigues forment ou non un tout. Les séries du corpus (voir liste en annexe) sont françaises (17), américaines (16), britanniques (2) et allemande (1). Nous avons arrêté les comptes en juin 2014, mais plusieurs séries sont toujours en production en 2015. 
récurrents, sur l'ensemble des saisons diffusées jusqu'en juin 20146. On dispose ainsi d'une vue d'ensemble du casting à un moment donné et de son évolution dans le temps. La proportion de personnages féminins a peu évolué en six décennies : en 2010 on en trouve $40 \%$ dans notre corpus, et seulement $34 \%$ si l'on ne retient que les personnages policiers ${ }^{7}$.

On observe surtout une disparité de la structure par âge. Que ce soit dans les premiers travaux de 1953 ou sur un corpus de 2874 personnages des programmes américains antérieurs à 1974, les personnages féminins sont en moyenne plus jeunes ${ }^{8}$. Ces calculs ont pour inconvénient de faire attribuer subjectivement un âge approximatif par des observateurs. Pour mesurer plus finement l'âge, nous avons choisi de retenir celui des acteurs et actrices, qui est précis, objectif et évolutif. On sait en effet, grâce à ChalvonDemersay notamment, à quel point le « héros de série télévisée » est un composite dont le personnage et l'interprète sont indissociables? Il est donc utile de combiner les données sur les personnages et sur leurs interprètes. Il ressort que les comédiennes sont toujours plus jeunes en moyenne. Lors de la première saison, l'écart moyen est de 6 ans, puis il se creuse de saison en saison, car le turn over du casting touche moins les hommes que les femmes.

Ce qui frappe n'est pas seulement la moyenne mais la structure asymétrique. Alors qu'on trouve des comédiens de tous les âges, de 25 ans jusqu'à plus de 70 ans, ce n'est pas le cas des comédiennes. Une minorité de ces dernières dépasse 45 ans, plus rarement encore 50 ans $^{10}$. Des comédiennes de moins de 50 ans peuvent les atteindre ou les dépasser si la série dure et qu'elles sont maintenues au casting, comme la plus longue série du corpus, Julie Lescant, que Véronique Genest a incarné de 36 ans à 58 ans. Sur les 171 actrices du corpus, seules 26 ont

6 Au total : 407 personnages principaux et récurrents, 298 saisons diffusées en France en juin 2014. Un tiers des séries du corpus compte au moins dix saisons.

7 Pour une analyse chiffrée plus approfondie, notamment de la répartition par sexe et par âge, Arbogast 2015.

8 Tedesco 1974.

9 Chalvon-Demersay 2011.

10 Jermyn 2013 a constaté la même absence des femmes de plus de 50 ans à la télévision anglaise, y compris hors émissions de fiction. 
atteint l'âge de 50 ans, soit $15 \%$ d'entre elles, alors que 38\% des acteurs ont eu ou dépassé cet âge (89 sur 236). Parmi ces 26 actrices, 16 d'entre elles avaient 50 ans au moins à leur prise de rôle, par exemple Marion Game (9 saisons greffière dans Boulevard du palais) ou Édith Scob (mère supérieure du couvent durant tout Scur Thérèse.com).

\section{En avoir ou pas (un compagnon)}

Parmi ces 26 cas, 20 personnages n'ont plus de compagnon ni de sexualité, voire pour certaines nonnes de Saur Thérèse n'en ont jamais eue. La cheffe du NCIS Los Angeles, jouée par Linda Hunt depuis ses 64 ans, a eu un amant agent secret dans un passé lointain, de même que Sœur Thérèse (Dominique Lavanant) est depuis longtemps divorcée de l'inspecteur joué par Martin Lamothe. S’il existe une image dominante des femmes de 50 ans ou plus, ce serait celle de femmes sorties de la sexualité. La détective Jo Danville des Experts Manhattan (dont Sela Ward prend le rôle à 54 ans) est mère divorcée et célibataire comme sa collègue de Las Vegas Catherine Willows (jouée par Marg Hengelberg de 42 ans à 53 ans), qui se consacre à sa fille pour conjurer un passé sulfureux, bien que le personnage finisse par avoir une relation avec un policier plus jeune (l'acteur Alex Carter a 6 ans de moins). Il a fallu dix saisons au casting des Experts, et le départ de personnages charismatiques, pour que Willows puisse retrouver une vie sexuelle, vers les 50 ans de son interprète. Cette trajectoire est rare, mais prouve que toute sortie n'est pas définitive.

D'autres parmi ces 26 personnages ont des partenaires, mais peu occupent des rôles de premier plan, épouses à la limite du faire-valoir (Jane Wymark jouant 13 saisons l'épouse de l'Inspecteur Barnaby, de 9 ans son aîné), ou dont le statut marital est si rarement évoqué qu'il passe inaperçu (la capitaine Gates du commissariat de Castle). Là encore, ce n'est qu'en durant plusieurs saisons que ce dernier personnage a gagné le droit d'avoir une vie personnelle évoquée en dialogue. En dehors de Willows, en couple avec un homme plus jeune, deux de ces personnages affichent une sexualité revendiquée. La commissaire Mercier des Bleus (Clémentine Célarié, 52 ans) renoue une liaison avec le capitaine Duval sous ses ordres (Jean-Michel Fête, 44 ans). Enfin Martha Rodgers, mère de l'écrivain Castle qui donne 
son nom à la série, 67 ans à la prise de rôle, est une célibataire très active dont le personnage sera décrit plus loin.

\section{Couples : l'art du grand écart}

Il serait tout à fait réalisable que les couples du petit écran réunissent des interprètes d'âges proches. Ce n'est pas du tout le cas. Dans notre corpus, 66 unions impliquent au moins un personnage principal ou récurrent. Dans seulement trois cas les interprètes sont né.e.s la même année. Il arrive que l'actrice soit la plus âgée du couple, d'un an (3 cas), deux, trois, six et sept ans $(1 \mathrm{cas})$, et jusqu'à huit ans $(2 \mathrm{cas})^{11}$. Mais l'ensemble est fortement asymétrique, puisque le cas général est que l'homme est plus âgé (54 fois sur 66). Les écarts d'âge sont beaucoup plus importants dans ce sens : dans 23 de ces unions il dépasse huit ans. Dans les quatre cas les plus extrêmes, il atteint ou dépasse vingt ans. Des écarts aussi importants sont peu fréquents dans le monde social. Certes les hommes y sont en moyenne un peu plus âgés que leur compagne, mais d'un peu plus de deux ans seulement. Les écarts importants sont rares, bien qu'ils soient plus nombreux qu'auparavant ${ }^{12}$ et plus fréquents dans les deuxièmes unions des hommes.

Trois traits caractérisent ces couples hétérosexuels. D’abord, la pression âgiste sur les femmes. Ensuite la possibilité offerte aux héros masculins d'avoir tous les âges. Enfin, l'écart d'âge parait bel et bien être une inégalité, au profit des hommes. C'est ce que Michel Bozon appelle «une domination par l'âge » ${ }^{13}$. Les structures d'âge asymétriques, les seuils du vieillissement plus bas pour les femmes, participent d'une valence différentielle des sexes ${ }^{14}$. La jeunesse des partenaires féminines est un élément de leur désirabilité sur le marché sexuel et matrimonial. Dans notre corpus, lorsque les comédien.ne.s ont le même âge, dans deux cas sur trois une autre forme de domination vient se glisser : l'homme est plus gradé. Dans le couple,

\footnotetext{
11 Concernant les couples dont la femme est la plus âgée, voir Bozon 1991.

12 Bozon 1990 ; Vanderschelden 2006.

13 Bozon 1990.

14 Soit un double mouvement de différenciation et de hiérarchisation des sexes, Héritier 1995.
} 
les formes de domination peuvent se combiner. Ainsi dans Un flic, le commissaire Schneider a une relation avec une lieutenante sous ses ordres (dont on ne connait que le prénom, Alex), et l'acteur a seize ans de plus que sa partenaire. Le capital social et économique d'un homme accroît en effet sa capacité à former une deuxième union avec une femme beaucoup plus jeune ${ }^{15}$. La différence d'âge est parfois si grande (26 ans dans Commissaire Brunetti) qu'elle pousse à s'intéresser à la place de l'excès et de l'exagération dans les rapports de genre représentés dans les séries.

\section{Quand la domination masculine déborde:}

la place de l'excès dans les rapports de genre

À en croire Todd Gitlin, qui a exploré longuement le fonctionnement des grandes chaînes de télévision, ce média est « une industrialisation de l'excès ${ }^{16}{ }^{16}$. Rien d'étonnant alors que les rapports de genre y soient outrés, plus contrastés que dans la vie sociale. Comment interpréter ces excès et quelle place leur accorder ? Raewyn Connell a théorisé la pluralité et la hiérarchie des masculinités et propose une typologie où le pivot est la masculinité hégémonique, de laquelle se distinguent des masculinités complices, subordonnées (ou dominées) et marginalisées ${ }^{17}$. Les masculinités " excessives », dans les classes populaires par exemple ou chez des hommes noirs ou latino-américains, sont déconsidérées par les détenteurs de l'hégémonie. L'excès est une forme inférieure dans la hiérarchie des masculinités.

À cet excès-là, répond la féminité «exagérée» (emphasiz̨ed femininity), forme hégémonique de la féminité qui répond aux attentes masculines. L'excès et l'exagération éclairent d'un jour nouveau le système d'asymétries de genre. Les différences d'âge importantes entre partenaires peuvent être lues sous cet angle, les écarts exagérés seraient alors un élément charnière de la domination masculine. Cette piste est prometteuse car la masculinité hégémonique s'accommode tant bien que mal du vieillissement, tandis que la féminité exagérée est par définition jeune dans son expression type (qui répond aux

\footnotetext{
15 Mignot $2010: 290$.

16 Gitlin $1994: 62$.

17 Connell 2014.
} 
attentes masculines). Un écart d'âge important pourra alors être interprété comme un excès constitutif des rapports de genre inégaux, entre les formes hégémoniques de masculinité et de féminité. Dans les séries, l'excès a-t-il pour effet de renforcer les inégalités de genre?

\section{Les femmes mûres ont-elles une vie sexuelle? Trois études de cas}

Puisque les séries comptent très peu de rôles de femmes d'âges mûrs, en examiner des exemples ne répond pas à un impératif de représentativité, mais à un enjeu de compréhension de ces situations minoritaires. On retiendra trois cas particuliers de femmes ayant une vie amoureuse après 50 ans, et qui en cela s'éloignent du cas général qu'on vient de présenter. Chacun permettra de tester la pertinence d'outils d'analyse des représentations de la sexualité des femmes de plus de 50 ans. Le premier personnage questionne l'importance d'avoir dépassé l'âge fertile. Le second pose la question de savoir si c'est l'humour qui rend acceptable la représentation de la sexualité des femmes. On cherchera ensuite comment les approches en termes d'hégémonie éclairent les comportements d'un troisième personnage. La théorie des scripts sexuels sera le dernier outil mobilisé pour comprendre ces situations, atypiques sinon transgressives.

\section{L'expertise sexuelle de toute une vie}

Pour les femmes, la période féconde prend fin autour de 45-50 ans, rarement après 50 ans ${ }^{18}$. C'est aussi à cet âge que la présence des femmes dans le casting connaît une chute spectaculaire. L'image négative des femmes âgées ${ }^{19}$ se combine avec les jugements dévalorisants sur les femmes ménopausées (devenues « hors d'âge »), plus d'une fois observés. Ces préjugés ont perduré dans les fictions audiovisuelles ${ }^{20}$. Comme l'a montré Paola Tabet pour de nombreuses sociétés, la sexualité est massivement organisée autour de la fécondabilité des femmes ${ }^{21}$. Celle des femmes plus âgées est donc peu pensée, encore moins représentée.

\footnotetext{
18 Ined 2010.

19 Héritier 1995 ; Théré 2013.

20 Bazzini et al. 1997.

21 Tabet 1998.
} 
Le premier exemple est tiré de Tell me you love me, série du câble américain d'une seule saison, diffusée en France sur TPS star. C'est donc un programme «de niche» destiné à un public de sériephiles ${ }^{22}$. Dans la série, plusieurs femmes en âge d'avoir des enfants ont une vie sexuelle et amoureuse très insatisfaisante. Ayant des difficultés à rester en couple, à avoir un enfant, ou à retrouver un amour conjugal noyé dans le quotidien d'une famille nombreuse, trois femmes (dont les comédiennes ont de 29 ans à 46 ans) viennent consulter le Dr May Foster, alias Jane Alexander (68 ans). May Foster vit, depuis 43 ans avec le même homme, un amour assumé au grand jour dès le premier épisode (baiser passionné, préliminaires sexuels).

La sexualité heureuse du personnage du Dr Foster propose une image rare à l'écran, qui, de plus, s'oppose à celle problématique de ses patientes plus jeunes. La fin de la période féconde n'est donc plus présentée comme la fin de la sexualité, mais comme le début d'un plaisir accru, libéré des contraintes de la fertilité. Au-delà de la ménopause, qui n'est pas dénigrée ici, c'est le regard sur la sexualité des femmes dans leur ensemble qui est original dans ce programme. Lorsque May Foster et son mari font l'amour, on voit entièrement nus ces deux corps âgés ('actrice a 68 ans et l'acteur 66), ce qui est inhabituel.

\section{Des seins sous étroite surveillance}

Jean-Claude Kaufmann a étudié les subtils codes non-écrits qui régissent les seins nus à la plage. Sous le discours égalitariste des plagistes, les jugements sont très normatifs. Les corps les moins sveltes, les moins jeunes, les seins pas assez menus et dont le balancement est donc visible, reçoivent la réprobation. Kaufmann décrit la construction d'un stigmate, dont l'exclusion du « sein vieux » est un aspect.

Car les seins nus accentuent la pression normative fondée sur la jeunesse et la beauté. Et elle ajoute pour les femmes un nouveau cercle d'exclusion (premier pas vers une mise à l'écart plus radicale) qui commence parfois à 35 ans ou 40 ans $^{23}$.

\footnotetext{
22 Donnat \& Pasquier 2011.

23 Kaufmann 1998 : 224.
} 
La plage est un espace public, extérieur, alors que la télévision entre dans le foyer : la relation qu'on entretient avec elle est intime ${ }^{24}$.

Y montrer un couple âgé en plein acte sexuel, et notamment une femme de 68 ans nue, tient de la transgression. Mais à bien y regarder, dans Tell me you love me le rapport à la nudité n'est pas si transgressif, puisque c'est la plus jeune et la plus svelte des femmes qu'on voit le plus entièrement nue et le plus souvent. Plus l'âge des actrices augmente et moins elles apparaissent nues à l'écran. Le degré de nudité, la fréquence et la durée des scènes de sexe, confirment que la jeunesse et la sveltesse sont un critère déterminant de la nudité acceptable.

\section{L'humour, pour faire passer la pilule de l'âge?}

On a souligné la rareté de rôles proposés à des femmes d'âge mûr. Les exceptions existent, comme dans Hot in Cleveland, série inédite en France, où Betty White endosse à 88 ans le rôle d'une logeuse délurée. Pour la journaliste Laura Bennett, le ressort comique de la série consiste à faire dire à la presque nonagénaire un chapelet de grivoiseries caricaturales ${ }^{25}$. Selon Beatriz Oria ${ }^{26}$, l'humour a partie liée avec la représentation télévisuelle de la sexualité des femmes. Analysant la série américaine Sex and the city, elle explique que c'est le traitement humoristique qui facilite, et même rend possible, de faire parler des femmes de sexe ouvertement, et d'en faire le cœur du propos. La série a pris fin avant qu'aucune actrice atteigne 50 ans (trois avaient moins de 40 ans, l'aînée 48 ans). Si l'humour est indispensable pour que des femmes d'âges intermédiaires assument librement leur sexualité, qu'en sera-t-il pour des comédiennes plus âgées?

\section{Martha Rodgers, ou la comédie du non-remariage}

Le deuxième cas étudié est la série Castle, tirée du corpus décrit plus haut. L'humour est très présent dans cette fiction qui mélange des éléments de comédie et de "drame» (un sous-genre baptisé dramedy).

\footnotetext{
24 Chalvon-Demersay 1999.

25 "These characters aren't actually exploring the sexuality of older women they're stunts to generate cheap laughs ", Bennett $2013: 6$.

26 Oria 2014 ; voir aussi Markle 2008.
} 
On trouve parmi les personnages récurrents Martha Rodgers, son fils qui tient le rôle-titre, et sa petite fille lycéenne. Susan Sullivan a 67 ans lorsque débute la série, où elle campe un personnage fantasque de mère et grand-mère peu conventionnelle. Sa vie amoureuse est importante dans la caractérisation du personnage, et il en est souvent question. Dès le premier épisode, lors d'une soirée mondaine elle repère un homme à son goût et se lance à sa conquête ${ }^{27}$. Elle fait régulièrement allusion à la brièveté de ses aventures : «Les hommes sont comme les auditions. Il y en a toujours une au coin de la rue». Cette présentation de soi est même un élément récurrent de ses échanges avec sa petite-fille, qu'elle taquine après un long appel à son petit-ami : «Je t'en prie, j’ai eu des histoires qui ont duré moins longtemps ».

Le piquant de ses répliques et la tonalité légère de la série contribuent à faire de ce personnage un pivot comique de la distribution. Grâce à ses mots d'esprit, on rit volontiers avec Martha Rodgers plutôt qu'à ses dépens. Le personnage a de nombreux traits positifs, mais on peut penser avec Oria que c'est la part de comédie qui facilite l'évocation de la sexualité d'une sexagénaire dans une série de grande écoute. Car les séries programmées sur une chaîne payante auteuriste ne sont pas celles diffusées en début de soirée sur les grandes chaînes gratuites. La programmation dessine une cartographie: dans les cases les plus exposées figurent les séries les plus consensuelles ${ }^{28}$, tandis que les représentations transgressives sont repoussées aux marges de la grille de diffusion.

\section{Female masculinity ou masculinité hégémonique?}

Les apports de Connell gagnent à être complétés par ce que Judith Halberstam appelle les «masculinités féminines ${ }^{29}$. Elle utilise ce concept pour penser les situations de «masculinité sans homme».

27 Elle est en compagnie de sa petite-fille et de son fils à qui elle dit : «Un instant mon chéri, mon détecteur de vieux-beau vient de sonner. Bingo! Pas d'alliance. Restez à distance les enfants, maman va à la pêche ».

28 «Least objectionable », selon la formule d'un haut responsable de chaîne américaine cité in Gitlin 1994 : 61.

29 Halberstam 1998. 
Pour des personnages policiers, fonction virile dans une institution fortement teintée de virilisme, l'angle de la masculinité s'impose. Se peut-il qu'une femme soit un exemple de masculinité hégémonique ? Ou complice?

La série anglaise Scott \& Bailey, inédite en France à ce jour, a pour personnages centraux deux policières, qui ont pour chef la Detective Chief Inspector Gill Murray. C’est la comédienne Amelia Bullmore, 47 ans lorsque la série débute, qui l'interprète. Mère divorcée, Gill Murray incarne la réussite professionnelle et jouit de la liberté sexuelle d'une célibataire de plus de 45 ans. Lors d'une discussion, elle glisse à l'officier Scott qu'elle a une aventure, avec un collègue beaucoup plus jeune et moins gradé. Scott lui rétorque sur le ton de la plaisanterie : «Oh, Gill Murray's got a toy boy! »30. Ultérieurement, Murray et une homologue ont une joute verbale amicale. Elle a le dernier mot en glissant froidement «I've got a toy boy ». Coucher avec un partenaire plus jeune est donc une performance positive, un trophée, de la même manière que pourrait le revendiquer un homme en position bégémonique. Que des femmes parlent de leur «toy boy», leur « joujou», est exceptionnel, voire transgressif. Néanmoins, l'appropriation des codes de la domination masculine par une femme reste une manière de la reproduire, ce qui tend à rejouer les inégalités statutaires à l'œuvre dans le monde social, plutôt qu'à en présenter une alternative. Dans une perspective de genre, la transgression apparente reste donc relative. L'épisode relaté ici n'est pas représentatif de la série dans son ensemble et des rapports de genre qui y sont dépeints, mais il mérite d'être pensé, jusque dans sa rareté.

Ces trois personnages correspondent à des situations minoritaires, on l'a dit. Aucune des trois ne considère qu'avoir une sexualité active ne serait "plus de leur âge ». En cela, elles introduisent de nouveaux « scripts » dans la fiction télévisée.

30 La phrase signifie davantage que « Gill Murray se tape un petit jeune », car « toy boy » ravale l'homme concerné au rang de jouet sexuel. 


\section{De nouveaux scripts sexuels?}

Dans un contexte de quasi-absence de représentations de femmes mûres sexuellement actives dans la fiction audiovisuelle, les trois exemples présentés relèvent de l'exception. Étant donnée cette rareté, il est difficile de considérer qu'il existe dans les séries un stéréotype des femmes de plus de 50 ans. S'il existe, il consisterait plutôt à montrer des femmes dépourvues de vie sexuelle ou sentimentale. C'est pourquoi des concepts forgés en référence aux stéréotypes, comme ceux d'Éric Macé, les rend malcommodes à manier dans les exemples étudiés ici. Il propose en effet de parler de «contre-stéréotype» lorsqu'un personnage prend directement le contrepied d'un cliché (ethnoracial, dans le cadre de ses travaux), et d'appeler «antistéréotype » une réappropriation subversive de marqueurs identitaires ${ }^{31}$. L'approche des « scripts sexuels »32, en revanche, parait s'appliquer à ces études de cas.

Dans la vie sociale, la sexualité n'a pas d'âge maximum : en 2006 les femmes de plus de 50 ans vivant en couple déclaraient à $90 \%$ avoir une activité sexuelle, une proportion en hausse régulière depuis les années 197033. Mais précisément, les représentations télévisuelles ne sont pas un reflet du monde social. Si banal devant l'écran, mais si rare à l'écran... L'écart entre les deux, lorsqu'il existe, dit quelque chose de l'acceptabilité sociale d'une situation, du moins aux yeux de personnes responsables de la création (responsables de chaînes, producteurs). Étant donné la force des représentations associées à la jeunesse, montrer la sexualité de femmes de 50 ans ou 70 ans n'est pas une simple déclinaison, en plus âgée, des images et des scénarios majoritaires. Ces personnages proposent des scripts sexuels rares, sinon inédits.

31 «L'antistéréotype est défini par le fait qu'il constitue les stéréotypes comme la matière même de sa réflexivité, conduisant ainsi, en les rendant visibles, à déstabiliser les attendus essentialistes, culturalistes et hégémoniques de l'ethnoracialisation des minorités, mais aussi de la "normalité" blanche de la majorité », Macé $2007: 87$.

32 Gagnon 2008.

33 Bajos \& Bozon 2008. 
Les séries télévisées restent marquées par des traits observés depuis les années 1950 : les femmes y sont plus jeunes et moins nombreuses que les hommes. La différence d'âge joue un rôle pivot dans l'asymétrie des rapports de genre représentés à la télévision. Le rôle renforçateur de l'excès dans les inégalités de genre se traduit notamment par les écarts d'âge extrêmes entre partenaires.

En revanche, la représentation de la sexualité des femmes de plus de 50 ans est plus ambivalente. Innovante et, par certains aspects, transgressive, elle met en scène une sexualité non-reproductive et accorde une place importante à des femmes généralement exclues du marché sexuel dans ces fictions. Toutefois, que ce soit par l'humour qui en relativise la portée, par une hiérarchie maintenue dans les nudités acceptables, ou par l'appropriation d'une masculinité hégémonique, ces séries ne rompent jamais entièrement avec les inégalités de genre. Pour rares qu'ils soient, au point qu'il faille en chercher une partie hors des chaînes les plus regardées, les exemples présentés démontrent que les situations marginales éclairent la norme ${ }^{34}$, d'une façon complémentaire aux données quantitatives.

\section{Annexe}

\section{Liste des 36 séries du corpus}

\section{(années de première diffusion française en clair) :}

Adresse inconnue (France 3, 2008-2009); Affaires étrangères (TF1, 2010-); Alice Nevers - le juge est une femme (TF1, 2002-) ; Bones (M6, 2007-) ; Boulevard du palais (France 2, 1999-) ; Castle (France 2, 2010-) ; Cold case - affaires classées (France 2, 2007-2011) ; Commissaire Brunetti (France 3, 2006-2012) ; Commissaire Magellan (France 3, 2009-) ; Diane femme flic (TF1, 2003-2010) ; Enquêtes réservées (20092013); Esprits criminels (2006-); FBI portés disparus (2004-2010); Inspecteur Barnaby (France 3, 2001-) ; Inspecteur Frost (France 3, 1999-2010) ; Julie Lescaut (TF1, 1992-2014); Les bleus - premiers pas dans la police (M6, 2008-2010); Les Experts (TF1, 2001-); Les Experts : Manbattan (TF1, 2005-2014); Les Experts : Miami (TF1, 2003-2013); Lie to me (M6, 2010-2011); Marion Mazzano (France 2, 2010) ; Medium (M6, 2004-2010) ; Mentalist (TF1, 2010-) ; NCIS (M6, 2004-); NCIS - Los Angeles (M6, 2010-); New York section criminelle (TF1, 2003-2012); Numb3rs (M6, 2005-2010) ; Profilage (TF1, 2009-); RIS, police scientifique (TF1, 2006-) ; Section de recherches (TF1, 2006-) ; Scaur Thérèse.com (TF1, 2002-2011) ; Sur

34 Debest 2013. 
le fil (France 2, 2008-2010); Tango (France 2, 2010-); The closer : enquêtes prioritaires (France 2, 2006-2012); Un flic (France 2, 2007-2012).

\section{Bibliographie}

ARBOGaSt Mathieu, 2015, «De si jeunes femmes... Analyse longitudinale des écarts d'âges et des inégalités de genre dans les séries policières », Genre en séries, 1, p. 73-99.

Bajos Nathalie \& Michel Bozon, 2008, Enquête sur la sexualité en France, Paris, La Découverte.

Bazzini Doris G., McIntosh William D., Smith Stephen M., CoOK Sabrina \& Caleigh HARRIS, 1997, "The aging woman in popular film: underrepresented. unattractive, unfriendly, and unintelligent », Sex roles, 36/7-8, p. 531-543.

BenNeTt Laura, 2013, "Betty White is not a sex machine. Our culture's cruel obsession with dirty old women », The New Republic, June 10, 2013, p. 5-6.

BozON Michel, 1990, «Les femmes et l'écart d'âge entre conjoints : une domination consentie. I. Types d'union et attentes en matière d'écart d'âge », Population, 44/2, p. 327-360.

—, 1991, «Les femmes plus âgées que leur conjoint sont-elles atypiques? », Population, 46/1, p. 152-159.

Chalvon-Demersay Sabine, 1999, « La confusion des conditions. Une enquête sur la série télévisée Urgences», Réseaux, 95, p. 235-283.

—, 2011, «Enquête sur l'étrange nature du héros de série télévisée », Réseaux, 165, p. 181-214.

CONNELl Raewyn, 2014, Masculinités. Enjeux sociaux de l'hégémonie, sous la direction de Meoïn Hagège et Arthur Vuattoux, Paris, Éd. Amsterdam [traduction augmentée de Masculinities, University of California Press, 2005].

COULOMB-GulLY Marlène, 2012, «La fabrique du genre dans les médias : vers un état des lieux et des problématiques ", Sciences de la societé, 82, p. 3-14.

DEBEST Charlotte, 2013/1, "Quand les "sans-enfant volontaires" questionnent les rôles parentaux contemporains ", Annales de démographie historique, 125, p. 119-139.

Dominick Joseph R., 1979, «The portrayal of women in prime time, 1953-1977 », Sex Roles, 5/4, p. 405-411.

Donnat Olivier \& Dominique PASQuier, 2011, «Présentation. Une sériphilie à la française », Réseaux, 165, p. 9-19.

GaGNON John, 2008, Les Scripts de la sexualité. Essais sur les origines culturelles du désir, Paris, Payot.

GiTuIN Todd, 1994 (revised edition), Inside prime time, London, Routledge. 
Halberstam Judith, 1998, Female masculinity, Durham, Duke University Press.

Héritier Françoise, 1995, Féminin/Masculin, la pensée de la différence, Paris, Odile Jacob.

INED, 2010, À quel âge devient-on sterile?, fiche pédagogique.

Jermyn Deborah, 2013, « Past their prime time? women, ageing and absence on British factual television », Critical Studies in Television, 8/1, p. 73-90.

KaUfmann Jean-Claude, 1998, Corps de femmes, regards d'bommes, sociologie des seins nus, Paris, Pocket.

LauZen Martha M. et David M. Dozier, 2005, « Recognition and Respect Revisited: Portrayals of Age and Gender in Prime-Time Television ", Mass communication and society, 8/3, p. 241-256.

LinColn Anne E. \& Michael Patrick AlLen, 2004, « Doublejeopardy in Hollywood: age and gender in the careers of film actors, 1926-1999», Sociological Forum, 19/4, p. 611-631.

MACÉ Éric, 2007, «Des "minorités visibles" aux néostéréotypes. Les enjeux des régimes de monstration télévisuelle des différences ethnoraciales », Journal des anthropologues, Hors-série.

MARKLE Gail, 2008, "“Can women have sex like a man?”: sexual scripts in Sex and the City", Sexuality \& Culture, 12/1, p. 45-57.

Mignot Jean-François, 2010, "L'écart d'âge entre conjoints ", Revue française de sociologie, 51/2, p. 281-320.

Oria Beatriz, 2014, "What's Love Got to Do With It? Sex and the City's Comic Perspective on Sex », Journal of Popular Culture, 47/2, p. 381-397.

TABET Paola, 1998, «Fertilité naturelle, reproduction forcée », in La Construction sociale de l'inégalité des sexes. Des Outils et des corps, Paris, L'Harmattan, p. 77-180.

TedesCo Nancy, 1974, "Patterns in prime time», Journal of communication, 24/2, p. 119-124.

THÉRÉ Christine, 2013, «Histoire de la représentation de la ménopause », Séminaire de recherche de l'unité Démographie, genre et société, «âge et cours de la vie : une construction genrée », Ined, 14 novembre.

VANDERSChElDEN Mélanie, 2006, «L'écart d'âge entre conjoints s'est réduit », Insee Première, ${ }^{\circ} 1073$.

Waxman Sharon, 2015, «Maggie Gyllenhaal on Hollywood ageism », The Wrap, 20 mai, http://www.thewrap.com/maggie-gyllenhaal-on-hollywood-ageism-i-wastold-37-is-too-old-for-a-55-year-old-love-interest/ [consulté le 12 juillet 2015] 
